\title{
Arginase Inhibitor in the Pharmacological Correction of Endothelial Dysfunction
}

\author{
Mihail V. Pokrovskiy, ${ }^{1}$ Mihail V. Korokin, ${ }^{2}$ Svetlana A. Tsepeleva, ${ }^{3}$ \\ Tatyana G. Pokrovskaya, ${ }^{1}$ Vladimir V. Gureev, ${ }^{2}$ Elena A. Konovalova, ${ }^{3}$ \\ Oleg S. Gudyrev, ${ }^{2}$ Vladimir I. Kochkarov, ${ }^{1}$ Liliya V. Korokina, ${ }^{3}$ \\ Eleonora N. Dudina, ${ }^{3}$ Anna V. Babko, ${ }^{3}$ and Elena G. Terehova ${ }^{3}$ \\ ${ }^{1}$ Department of Pharmacology and Pharmaceutical Sciences, Belgorod State University, 85 Pobeda Street, Belgorod 308015, Russia \\ ${ }^{2}$ Research Institute of Ecological Medicine, Kursk State Medical University, 3 Karl Marx Street, Kursk 305041, Russia \\ ${ }^{3}$ Department of Pharmacology, Kursk State Medical University, 3 Karl Marx Street, Kursk 305041, Russia
}

Correspondence should be addressed to Mihail V. Pokrovskiy, mpokrovsky@yandex.ru

Received 15 February 2011; Accepted 25 April 2011

Academic Editor: Zafar Israili

Copyright (c) 2011 Mihail V. Pokrovskiy et al. This is an open access article distributed under the Creative Commons Attribution License, which permits unrestricted use, distribution, and reproduction in any medium, provided the original work is properly cited.

This paper is about a way of correction of endothelial dysfunction with the inhibitor of arginase: L-norvaline. There is an imbalance between vasoconstriction and vasodilatation factors of endothelium on the basis of endothelial dysfunction. Among vasodilatation agents, nitrogen oxide plays the basic role. Amino acid L-arginine serves as a source of molecules of nitrogen oxide in an organism. Because of the high activity of arginase enzyme which catalyzes the hydrolysis of L-arginine into ornithine and urea, the bioavailability of nitrogen oxide decreases. The inhibitors of arginase suppress the activity of the given enzyme, raising and production of nitrogen oxide, preventing the development of endothelial dysfunction.

\section{Introduction}

The reasons and pathogenesis of cardiovascular diseases have always been the object of steadfast attention as the given pathology remains a principal cause of death rate of people. The list of the most frequent diseases of heart and vessels include ischemic illness of heart, arterial hypertension, cerebrovascular diseases. In the pathogenesis of these disorders, the basic role belongs to endothelial dysfunction [1].

Endothelial dysfunction is the first stage of development of heart and vessel illnesses. It is known that endothelium is capable of excreting factors of vasodilatation which relax smooth muscles of a vascular wall and lead to vasodilatation. On the other hand, there are endothelium factors of vasoconstriction. As a whole, on endothelial dysfunction, an imbalance between these factors production and vasoconstriction agents' prevalence takes place [2]. In addition endothelial dysfunction, which can lead to the breakdown of the blood-brain barrier and impair cerebral autoregulation and prothrombotic changes, is believed to be important in mediating leukoaraiosis. The normal cerebral endothelium plays a crucial role in the regulation of cerebral blood flow and autoregulation and in the blood-brain barrier. In addition, in health, it presents an anticoagulant phenotype to blood. Upon stimulation by numerous agents, the endothelium undergoes changes that allow it to participate in the inflammatory response; this is known as endothelial cell activation (ECA) [3]. One of the changes of ECA is increased vascular permeability, and it is thought that the entry of serum proteins into the vascular wall and perivascular neural parenchyma may produce toxic effects [4].

Endothelial dysfunctions may also give rise to molecular events involving a shift in the $\mathrm{O}(2)$ and $\mathrm{CO}(2)$ trafficking system in the red blood cells, which will result in special complex microcirculation disturbances in the white matter of the brain [5]. Also slight chronic hypoperfusion or an endothelial dysfunction may lead indirectly to a malfunction of the molecular crosstalk between the nucleus and the mitochondria [6]. 
The basic vasodilatation agent is considered to be a molecule of nitrogen oxide (NO). Differently, endothelial dysfunction is infringement of NO synthesis. In normally functioning endothelium, there is a constant $\mathrm{NO}$ production with the help of endothelial NO synthase (eNOS) from L-arginine. It refers to group of semi-irreplaceable amino acids and plays an important role in organism vital activity. Not so long ago it has been established that L-arginine is the predecessor of the NO possessing a wide spectrum of bioregulation influences. NO production infringement on endothelial dysfunctions is associated with reduction of availability of L-arginine stocks for eNOS, acceleration of NO metabolism, or a combination of both [7]. Thus, the basic source of NO is L-arginine which arrives in an organism with food. Because of the high activity of arginase-the enzyme destroying L-arginine in a mucous membrane of thin intestine, $40 \%$ of arginine arriving with food is destroyed in the course of absorption, and its remaining quantity arrives into a portal vein. Accepting the fact that $90 \%$ of L-arginine is connect with protein, it is possible to consider that only $50 \%$ of alimentary arginine goes into system circulation. The arginase is an enzyme of urea cycle that hydrolyzes Larginine to ornithine and urea. There are two isoforms of this enzyme. Arginase I is constitutive, and "extrahepatic" arginase (arginase II) is induced in vessel endothelium cells by lipopolysaccharides and interferon.

An other way of L-arginine catabolism proceeds with the formation of $\mathrm{NO}$ and citrulline. This process is catalyzed by another enzyme-NO synthase (NOS)—which exists in three isoforms: two constitutive, endothelial (eNOS) and neuronal (nNOS), and one induced (iNOS). They carry out the joining of molecular oxygen to nitrogen atom from terminal guanidine group of L-arginine. In the regulation of cardiovascular system, eNOS plays a leading role. Arginase and NOS compete for a common substratum-L-arginine. However, activity of arginase exceeds NOS activity by thousand times. Hence, basic part of L-arginine turns into ornithine and urea, thereby creating NO deficiency. In turn the lack of NO, as already has been mentioned earlier, leads to the development of endothelial dysfunction and to an increase of cardiovascular pathology development risk.

Thus, there is obviously a necessity of suppressing high activity of arginase the decrease to risk and frequency of development of illnesses of heart and vessels. With that aim now arginase inhibitors of substances of a natural origin [8] are investigated. Among substances of this group, Lnorvaline attracts the greatest interest, being nonselective inhibitor of arginase which is able to suppress activity of given enzyme, to raise endogenous stocks of L-arginine, and also to increase production of $\mathrm{NO}$, promoting normal functioning of vessels endothelium [9].

The purpose of the present research was studying L-norvaline endothelium-protective properties in L-NAME- and hyperhomocysteinemin-induced endothelial dysfunction.

\section{Materials and Methods}

Experiments were led on white rat males of Wistar line in mass of 200-250 grams.
NO synthase blocker, N-nitro-L-arginine methyl ester (L-NAME), was inducted intraperitoneally (i.p.) in a dose of $25 \mathrm{mg} / \mathrm{kg} /$ day, once a day for 7 days. For simulation of hyperhomocysteinemia, an amino acid methionine was given intragastrically in a dose of $3 \mathrm{~g} / \mathrm{kg} /$ day once a day for 7 days. A solution of methionine is intragastrically introduced ex tempore with polysorbate Tween- 80 and $1 \%$ starch solution. The data gained from intragastric introductionof an equivalent amount of polysorbate solution Tween- 80 was used as control.

L-norvaline was given i.p. in a dose of $10 \mathrm{mg} / \mathrm{kg}$ once a day for 7 days.

Animals have been partitioned into groups, each including 10 rats: (1) intact, (2) Tween-80; (3) L-NAME, (4) methionine, (5) L-NAME + L-norvaline $10 \mathrm{mg} / \mathrm{kg}$, and (6) methionine + L-norvaline $10 \mathrm{mg} / \mathrm{kg}$.

On the day 8 from the initiation of experiments under anaesthetic (chloral hydrate $300 \mathrm{mg} / \mathrm{kg}$ ), a catheter in the left carotid artery for recording of indexes of blood pressure (BP) was entered. Bolus introduction of pharmacological agents was made into a femoral vein. Hemodynamic indexes were the systolic arterial pressure (SAP), a diastolic arterial pressure (DAP), and cardiac contraction rate metered continuously with a hardware-software complex "Biopac". Besides BP measuring, a series of the functional trials was led in introduced succession: (1) endotheliumdependent vasorelaxation test (intravenous entering of a solution of acetylcholine $(\mathrm{AH})$ in a dose of $40 \mathrm{mkg} / \mathrm{kg}$ ); (2) endothelium-independent vasorelaxation test (intravenous entering of a solution of sodium nitroprusside (NP) in a dose of $30 \mathrm{mkg} / \mathrm{kg}$ ) [10-12].

Level of endothelial dysfunction in the experimental animals and also the level of its correction by researched drugs were valued on coefficient of endothelial dysfunction (CED). This coefficient risobtained by the following formula: $\mathrm{CED}=\mathrm{SBP}_{\mathrm{NP}} / \mathrm{SBP}_{\mathrm{AH}}$, where $\mathrm{SBP}_{\mathrm{NP}}$ is the area of triangle above a $\mathrm{BP}$ recovery curve at a functional test with NP entering and $\mathrm{SBP}_{\mathrm{AH}}$ is the area of triangle above a $\mathrm{BP}$ recovery curve at a functional test with $\mathrm{AH}$ entering. Points of a smaller cathetus of this triangle are the points of BP before the test and a point of maximum reduction of a $\mathrm{BP}$, and the bigger cathetus is the time of BP restoration [10-12].

The results were expressed as the mean $(\mathrm{M}) \pm$ the standard error of mean $(\mathrm{m})$. Differences were considered significant at $P<.05$.

\section{Results}

NOS blockade caused by a seven-day introduction of LNAME led to an arterial hypertension (SAP: 190,3 \pm 6,7 mm hg, DAP: $145,0 \pm 3,9 \mathrm{~mm} \mathrm{hg}$ ). Simultaneous LNAME and L-norvaline introduction did not lead to a decrease in indicators of BP reference values (Table 1). Daily introduction of methionine under the designated scheme did not cause authentic change of arterial pressure (Table 2). Introduction of L-norvaline i.p. also did not influence indicators of haemodynamics (Table 1).

For objectification of endothelial dysfunction correction estimations arising on modelling of deficiency of NO by 
TABLE 1: Dynamics of blood pressure indicators on modeling of NO deficiency and its correction with L-norvaline $(\mathrm{M} \pm \mathrm{m}, n=10)$.

\begin{tabular}{|c|c|c|c|c|c|}
\hline Groups of animals & Functional tests & SAP, mm hg & DAP, mm hg & Area of vascular reaction & CED \\
\hline \multirow{3}{*}{ Intact } & Before test & $137,7 \pm 3,7$ & $101,9 \pm 4,3$ & & \multirow{3}{*}{$1,1 \pm 0,1$} \\
\hline & $\mathrm{AH}$ & $84,3 \pm 4,5$ & $38,7 \pm 2,8$ & $1268,0 \pm 74,8$ & \\
\hline & NP & $83,0 \pm 3,7$ & $42,1 \pm 4,4$ & $1375,3 \pm 93,7$ & \\
\hline \multirow{3}{*}{ L-NAME $25 \mathrm{mg} / \mathrm{kg}$} & Before test & $190,3 \pm 6,7^{*}$ & $145,0 \pm 3,9^{*}$ & & \multirow{3}{*}{$5,4 \pm 0,6^{*}$} \\
\hline & $\mathrm{AH}$ & $110,6 \pm 5,2^{*}$ & $82,8 \pm 6,6^{*}$ & $695,3 \pm 87,6^{*}$ & \\
\hline & NP & $88,7 \pm 4,7$ & $50,8 \pm 4,2$ & $3322,7 \pm 116,7^{*}$ & \\
\hline \multirow{3}{*}{ L-NAME $25 \mathrm{mg} / \mathrm{kg}$ + L-norvaline $10 \mathrm{mg} / \mathrm{kg}$} & Before test & $180 \pm 4,7^{*}$ & $144,6 \pm 10,2^{*}$ & & \multirow{3}{*}{$2,1 \pm 0,2^{* *}$} \\
\hline & $\mathrm{AH}$ & $106,7 \pm 4,9^{*}$ & $56,1 \pm 1,8^{*}$ & $1360,6 \pm 126,9 *$ & \\
\hline & $\mathrm{NP}$ & $129,3 \pm 5,1^{* *}$ & $64,6 \pm 2,5^{* *}$ & $2827,2 \pm 429,1^{* *}$ & \\
\hline
\end{tabular}

${ }^{*} P<.05$ in comparison with intact group of animals; ${ }^{* *} P<.05$ in comparison with L-NAME group.

TABLE 2: Influence of methionine and L-norvaline on indicators of haemodynamics and coefficient of endothelial dysfunction in modeling of the homocysteine-induced NO deficiency $(\mathrm{M} \pm \mathrm{m}, n=10)$.

\begin{tabular}{lccccc}
\hline Groups of animals & Functional tests & SAP, mm hg & DAP, mm hg & Area of vascular reaction & CED \\
\hline & Before test & $129,2 \pm 4,3$ & $82,4 \pm 5,9$ & & $1124,2 \pm 63,7$ \\
Tween-80 & AH & $74,1 \pm 2,9$ & $39,4 \pm 3,1$ & $1011,8 \pm 94,6$ \\
& NP & $67,2 \pm 5,1$ & $42,9 \pm 5,4$ & \\
Methionine $3 \mathrm{~g} / \mathrm{kg}$ & Before test & $118,9 \pm 10,1$ & $76,6 \pm 7,2$ & \\
& AH & $80,1 \pm 2,9$ & $41,4 \pm 2,3$ & $854,6 \pm 61,4^{*}$ \\
& NP & $72,3 \pm 6,7$ & $45,9 \pm 4,3$ & $2820,2 \pm 210,4^{*}$ \\
Methionine $3 \mathrm{~g} / \mathrm{kg}+$ L-norvaline $10 \mathrm{mg} / \mathrm{kg}$ & Before test & $129,4 \pm 2,8^{* *}$ & $72,6 \pm 5,4^{* *}$ & \\
& AH & $91,0 \pm 3,1$ & $47,1 \pm 1,8^{* *}$ & $920,9 \pm 7,8^{*}$ \\
& NP & $123,8 \pm 4,5$ & $63,4 \pm 5,0^{* *}$ & $1214,7 \pm 128,4^{* *}$ \\
\hline
\end{tabular}

${ }^{*} P<.05$ in comparison with intact group of animals; ${ }^{* *} P<.05$ in comparison with metionine group.

L-NAME and methionine introduction, the special coefficient of endothelial dysfunction (CED) [12] is applied in our laboratory. It characterizes the endothelial dysfunction degree.

In each animal in each group, CED was measured. After modeling of NOS blockade, In the group of animals receiving L-NAME, CED was equal to $5,4 \pm 0,6$ while in group of intact animals CED was $1,1 \pm 0,1$. In the animals receiving L-norvaline against introduction of L-NAME, CED was $2,1 \pm 0,2$, coming a value close to that group of intact animals (Table 1). In animals receiving methionine, CED was $3,3 \pm 0,3$. In the animals receiving Tween- 80 CED was equal to $0,9 \pm 0,2$, and in animals receiving L-norvaline against methionine, CED was $1,4 \pm 0,1$ (Table 2).

\section{Discussion}

In conditions of normally functioning endothelium, the balance between vasoconstriction and vasodilatation factors is supported. The basic vasodilatation agent in endothelium is NO. Infringement of the given balance leads to development of endothelial dysfunction. L-arginine serves as a source of $\mathrm{NO}$ in a cell. Inhibitors of arginase, suppressing activity of the given enzyme, promote NO biosynthesis increase, preventing the development of endothelial dysfunction. Application of L-norvaline promotes suppression of activity

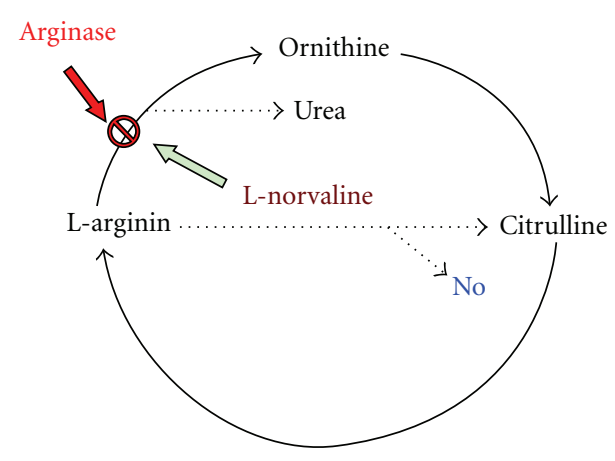

FIGURE 1: The mechanism of action of arginase inhibitors.

of arginase enzyme which allows for a raise in L-arginine stocks. Expressed endothelium-protective action is provided first of all with increase in stocks of endogenous L-arginine by infringement of its hydrolysis into ornithine and urea (Figure 1), which proves to be true by obvious decrease of CED in animals. In connection with the aforesaid, it is possible to speak about endothelium-protective action of the given group of substances and the prospect for their combined application with L-arginine and the preparations traditionally applied for cardiovascular disease treatment. 


\section{Conclusion}

The present research shows expressed endothelium-protective property of arginase inhibitor, L-norvaline, characterized by decrease of coefficient of endothelial dysfunction and the approached its application to a group of intact animals. In other words, L-norvaline prevents the development of systemic endothelial dysfunctions in L-NAME- and methionine-induced NO deficiency.

\section{References}

[1] G. S. Sainani and V. G. Maru, "Role of endothelial cell dysfunction in essential hypertension," Journal of Association of Physicians of India, vol. 52, pp. 966-969, 2004.

[2] A. L. Miller, "The effects of a sustained-release L-arginine formulation on blood pressure and vascular compliance in 29 healthy individuals," Alternative Medicine Review, vol. 11, no. 1, pp. 23-29, 2006.

[3] B. J. Hunt and K. M. Jurd, "Endothelial cell activation," British Medical Journal, vol. 316, no. 7141, pp. 1328-1329, 1998.

[4] H. Tomimoto, I. Akiguchi, H. Wakita, A. Osaki, M. Hayashi, and Y. Yamamoto, "Coagulation activation in patients with Binswanger disease," Archives of Neurology, vol. 56, no. 9, pp. 1104-1108, 1999.

[5] Z. Szolnoki, "Chemical events behind medical chemistry offers new insight into a specific microcirculation disturbance in the brain (a chemical approach to a frequent cerebral phenotype," Current Medicinal Chemistry, vol. 14, no. 9, pp. 1027-1036, 2007.

[6] Z. Szolnoki, "Pathomechanism of leukoaraiosis: a molecular bridge between the genetic, biochemical, and clinical processes (a mitochondrial hypothesis)," NeuroMolecular Medicine, vol. 9, no. 1, pp. 21-34, 2007.

[7] M. Kelm, "The L-arginine-nitric oxide pathway in hypertension," Current Hypertension Reports, vol. 5, no. 1, pp. 80-86, 2003.

[8] N. N. Huynh, E. E. Harris, J. F. P. Chin-Dusting, and K. L. Andrews, "The vascular effects of different arginase inhibitors in rat i/ated aorta and mesenteric arteries," British Journal of Pharmacology, vol. 156, no. 1, pp. 84-93, 2009.

[9] X. F. Ming, A. G. Rajapakse, J. M. Carvas, J. Ruffieux, and Z. Yang, "Inhibition of S6K1 accounts partially for the antiinflammatory effects of the arginase inhibitor L-norvaline," BMC Cardiovascular Disorders, vol. 9, article 12, 2009.

[10] M. V. Pokrovskil, T. G. Pokrovskaya, V. I. Kochkarov, and E. B. Artyushkova, "Endothelioprotective properties of L-arginine on a nitric oxide deficiency model," The Experimental and Clinical Pharmacology, vol. 71, no. 2, pp. 29-31, 2008.

[11] M. V. Pokrovskii, V. I. Kochkarov, T. G. Pokrovskaya et al., "Comparative study of potential endothelioprotectors and impaza in modeled nitric oxide defi ciency," Bulletin of Experimental Biology and Medicine, vol. 148, no. 3, pp. 514$517,2009$.

[12] M. V. Pokrovskiy, T. G. Pokrovskaya, and V.I. Pokrovskaya, The patent. 2301015 Russian Federation, MPK $^{7}$ A61B 5/02.- - no. 2005113243/14. 


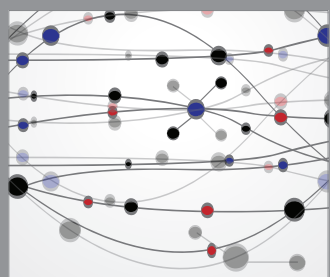

The Scientific World Journal
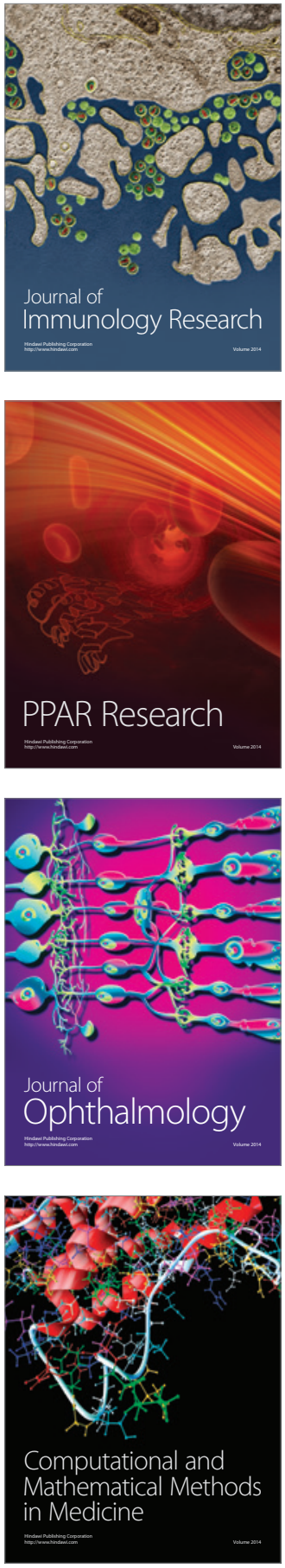

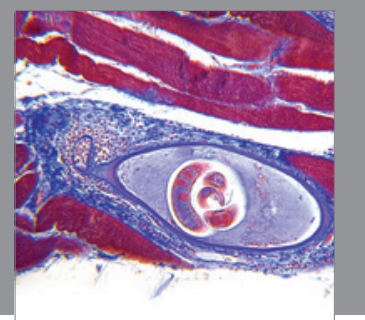

Gastroenterology

Research and Practice
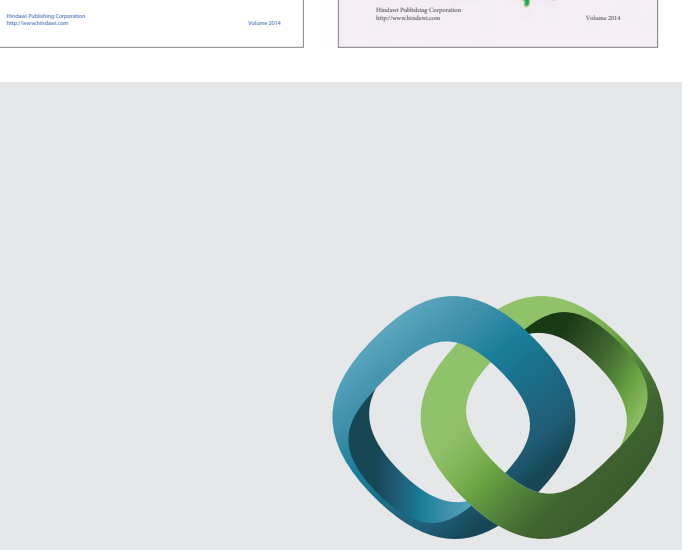

\section{Hindawi}

Submit your manuscripts at

http://www.hindawi.com
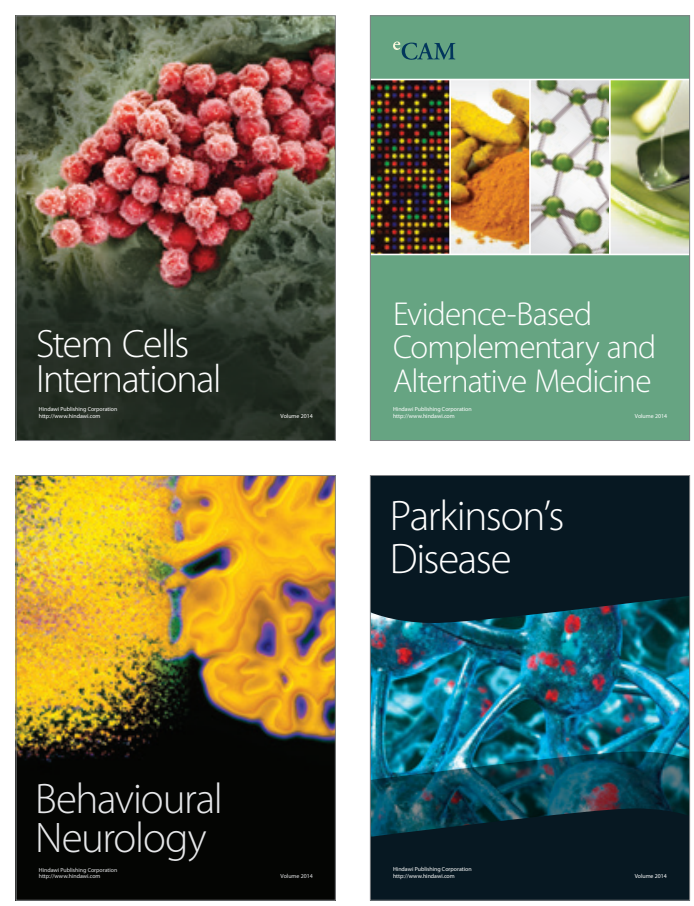

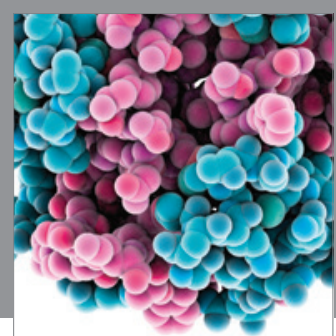

Journal of
Diabetes Research

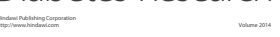

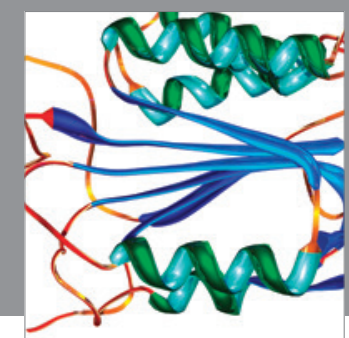

Disease Markers
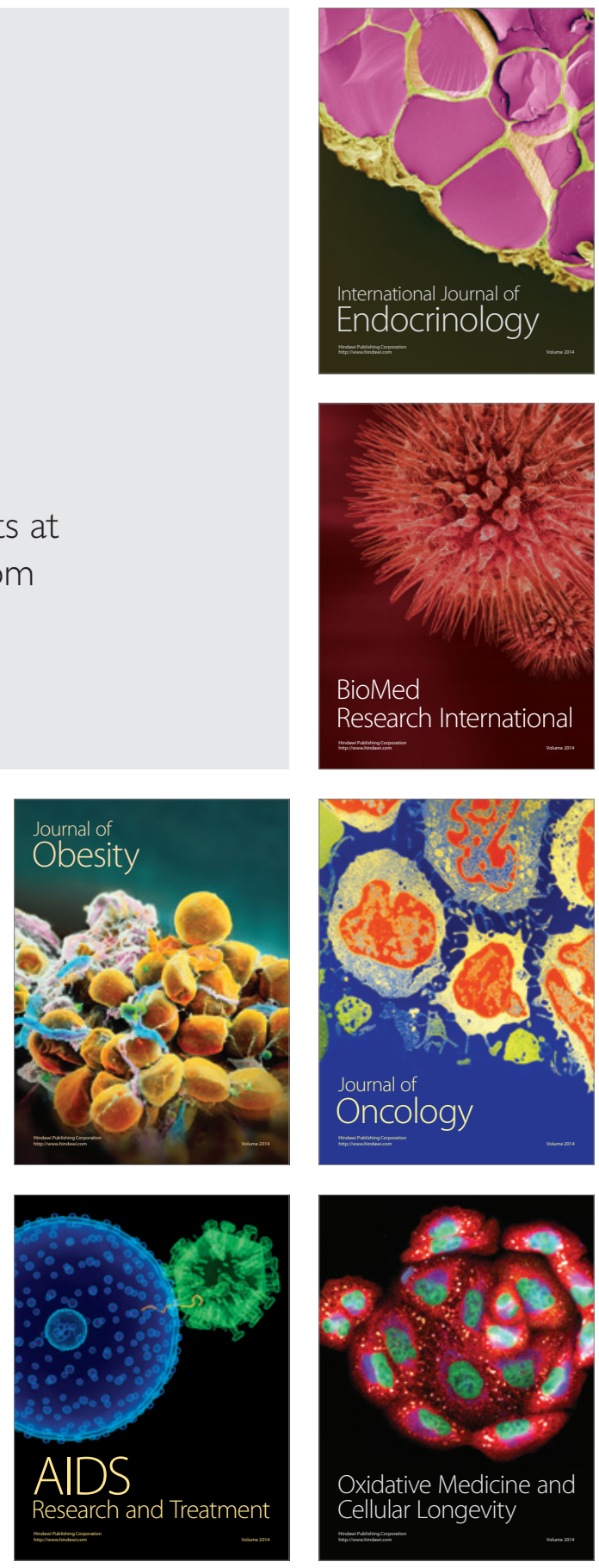